\title{
Delayed Replantation of Avulsed Permanent Incisor with 16 H Extra- Oral Time
}

Raja Belgacem $^{1 *}$, Farah Chouchène ${ }^{2}$, Fatma Masmoudi $^{3}$, Ahlem Baaziz $^{4}$, Fethi Maatouk ${ }^{5}$, Hichem Ghedira $^{6}$

${ }^{1}$ Resident in Pediatric Dentistry, Faculty of Dental Medicine of Monastir Tunisia, University of Monastir. Dento-Facial Biological and Clinical Approach Laboratory LR12ES10, Monastir 5000, Tunisia

${ }^{2}$ Assistant Professor in Pediatric Dentistry, Faculty of Dental Medicine of Monastir Tunisia, University of Monastir Dento-Facial Biological and Clinical Approach Laboratory LR12ES10, Monastir 5000, Tunisia

${ }^{3-6}$ Professor in Pediatric Dentistry, Faculty of Dental Medicine of Monastir Tunisia, University of Monastir Dento-Facial Biological and Clinical Approach Laboratory LR12ES10, Monastir 5000, Tunisia

$\begin{array}{cl}\text { Article History } & \begin{array}{l}\text { Abstract: Tooth avulsion in permanent dentition constitutes a dental emergency and } \\ \text { immediate replantation of the avulsed tooth is often considered as the treatment of }\end{array} \\ \text { Received: } 13.11 .2020 & \text { choice. This case report describes the management of a 13-year old boy with avulsed } \\ \text { Accepted: } 24.11 .2020 & \text { maxillary central incisor. The avulsed tooth was kept in milk for } 16 \text { hours from the } \\ \text { Published: } 28.11 .2020 & \begin{array}{l}\text { moment of trauma until its replantation. Extra-oral endodontic treatment was carried } \\ \text { out before replantation and splinting of the avulsed tooth was done with a semi rigid } \\ \text { splint for 4 weeks. The replanted tooth remained in a stable functional position and did } \\ \text { not reveal clinical or radiological symptoms. After 18 months follow-up period, a } \\ \text { Journal homepage: }\end{array} \\ \text { replacement root resorption was radiologically diagnosed. }\end{array}$

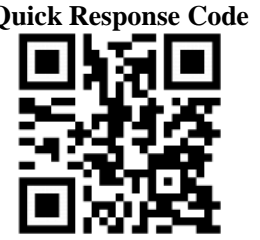

Keywords: Tooth avulsion, permanent dentition, replantation, root resorption.

Copyright () 2020 The Author(s): This is an open-access article distributed under the terms of the Creative Commons Attribution 4.0 International License (CC BY-NC 4.0) which permits unrestricted use, distribution, and reproduction in any medium for non-commercial use provided the original author and source are credited.

\section{INTRODUCTION}

Tooth avulsion is defined as a complete displacement of a tooth from its alveolar socket [1]. Considered as one of the most serious dental injuries, it is relatively infrequent in permanent dentition and ranges between $0.5 \%$ to $16 \%$ [2-4].

Tooth avulsion generally occurs in the maxilla, and the most commonly affected teeth are the maxillary central incisors $[5,6]$. Avulsion anterior tooth could not only lead to loss of function, but also will result in a negative impact on quality of life, producing psychological and esthetic problems [1, 4].

Appropriate emergency management is essential for attaining the best outcome after this injury and replantation is, in most situations, the treatment of choice but cannot always be carried out immediately. The factors which determine healing following replantation are essentially the length of extra-alveolar time and storage media $[5,6]$.

The aim of this report was to describe the management of an accidentally avulsed maxillary central incisor kept in milk for 16 hours from the moment of trauma until its replantation in a 13-year-old boy.

\section{Case Report}

A 13-year old boy was referred to the Pediatric Dentistry Department at the Dental Clinic of Monastir, Tunisia with a chief complaint of dental trauma sustained in the maxillary anterior region, associated with the avulsion of right central incisor.

The trauma occurred 16 hours ago while the child was playing in the school garden. The child had already been seen by the medical staff of the emergency unit of a local hospital who had reported no neurological damage or medical complications. His parents had kept the avulsed tooth in the milk from the moment of trauma and brought it to the clinic. No concomitant systemic disease was defined by the patient's parents.

The clinical examination showed laceration on upper lip and lower chin with avulsed maxillary right central incisor (Figure1). The patient had permanent 
dentition and his oral hygiene was poor. No other oral injury was detected clinically.

The periapical radiographs revealed no alveolar bone wall fracture or other hard tissue injuries (Figure 2).

The examination of avulsed tooth revealed that the root had a closed apex, and the root surface was covered with dirt and dried remnants of necrotic periodontal tissue. (Figure 3)

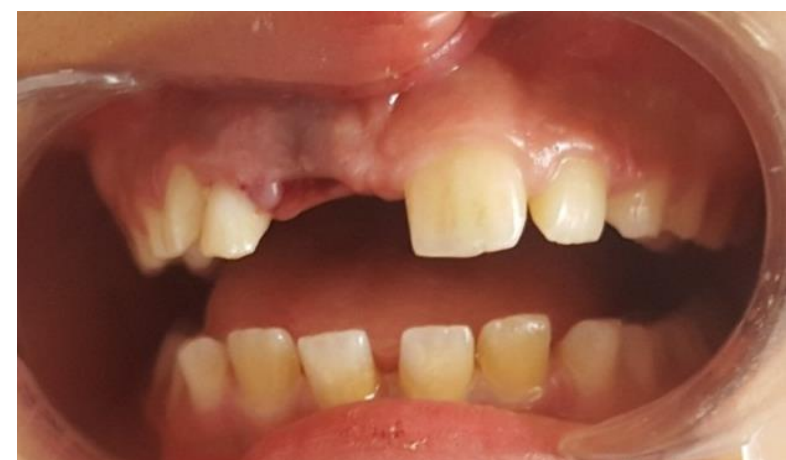

Fig-1: Preoperative intraoral photograph - Avulsion of the right upper incisor

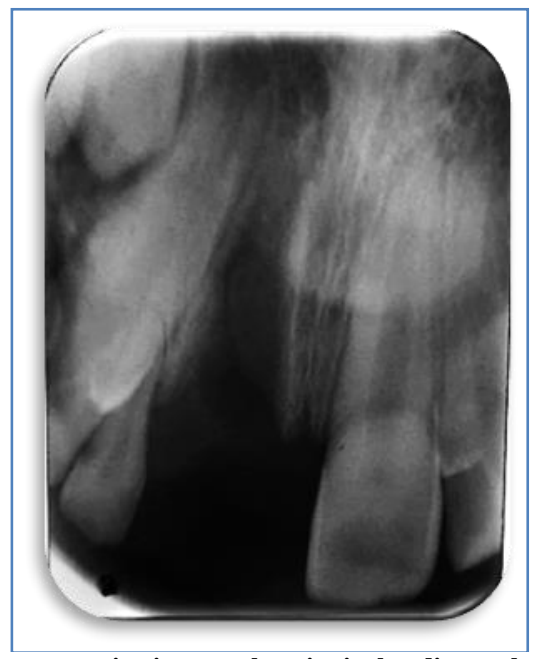

Fig-2: Pre-operative intraoral periapical radiograph showing extensive bone loss

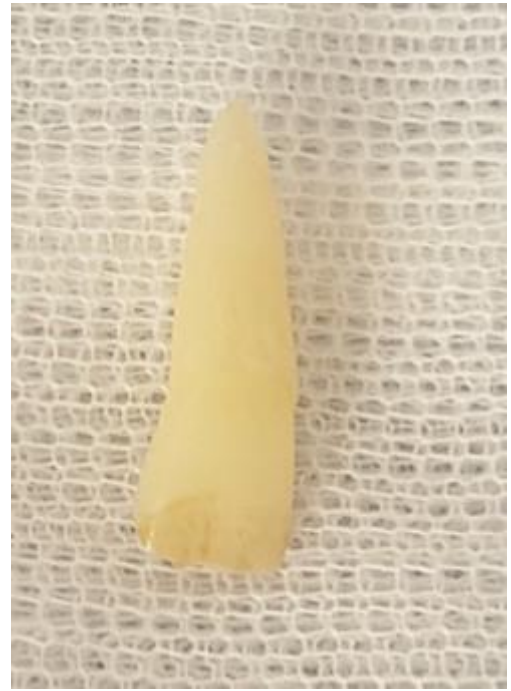

Fig-3: Clinical aspect of the avulsed tooth

After the examination, the treatment guideline for avulsed permanent teeth with closed apexes and prolonged extraoral time were followed.

Tooth was thoroughly washed in saline, and the root surface was cleaned carefully to remove necrotic and dried remnants of periodontal tissue. Then the tooth was treated with $2 \%$ sodium fluoride solution for 20 mins. Extra-oral endodontic treatment was carried out on the tooth (Figure 4): Access opening was done (Figure 4.a) and pulp was extirpated (Figure 4.b), manuel ProTaper file system was used to shape the canal (Figures 4.c). The canal was obturated using gutta percha cones (Figure 4.d).

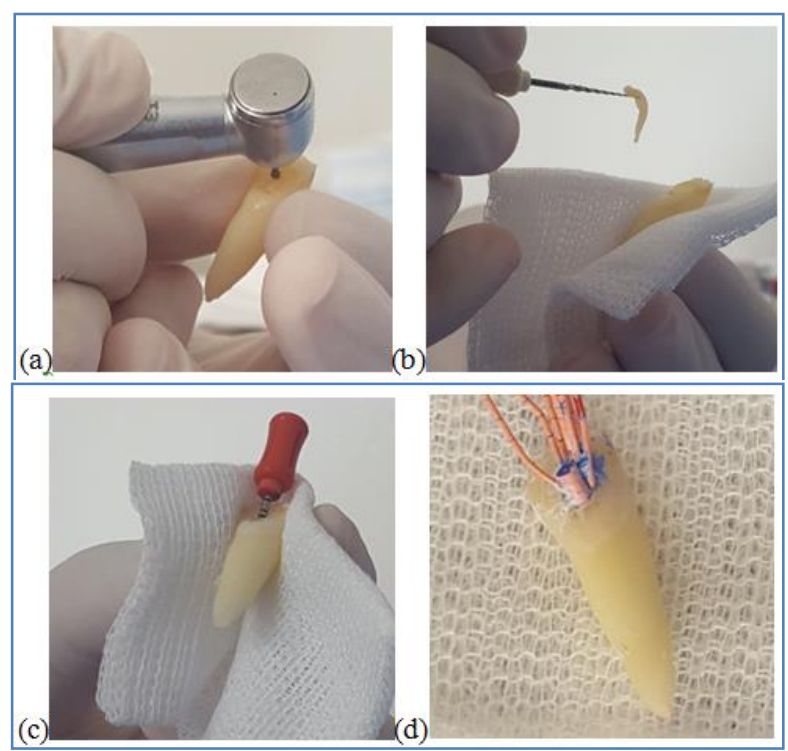

Fig-4: Extra-oral endodontic treatment

A: Access opening done with a carbide bur

B: pulp necrosis was extirpated

$\mathrm{C}$ : Cleaning and shaping of the canal done with manuel

ProTaper file system

D: canal was obturated with gutta percha cones 
Local anesthetic was administered (Figure 5), and socket was gently curetted to remove any coagulum, granulation or pathologic tissue and then irrigated with physiologic saline solution (Figure 6). The avulsed maxillary right central incisor was replanted slowly, with slight digital pressure (Figure 7).

A periapical radiograph was taken to ensure that the tooth had been correctly positioned in the socket (Figure 8). The tooth was splinted from first premolar to first premolar with a semi rigid splint for 4 weeks (Figure 9 and 10).

The patient was instructed about oral hygiene instructions and advised about a soft diet and the need to use a chlorhexidine mouth rinse during the stabilization period. A 7-day of systemic antibiotic therapy with amoxicillin at a dose of $2 \mathrm{~g}$ /day was prescribed.

The patient was reviewed after 4 weeks, and no clinical or radiological pathological changes were detected. The splinting wire was removed at this appointment.

The patient was seen again four weeks after replantation and 3 month, and then he was regularly kept under follow-up every 6 months. Clinical and radiographic controls were performed.

During the 12-month follow-up, clinical and radiographic examinations showed satisfactory functional and esthetic values for the avulsed tooth but some initial replacement root resorption was developed later (Figure 11).

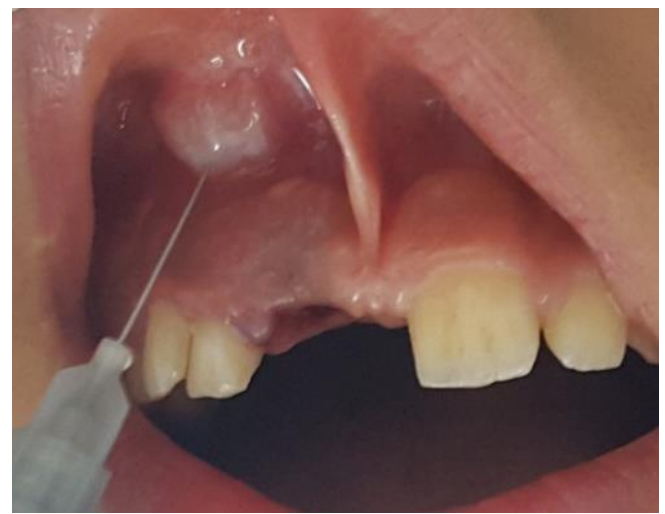

Fig-5: Local anesthesia administered

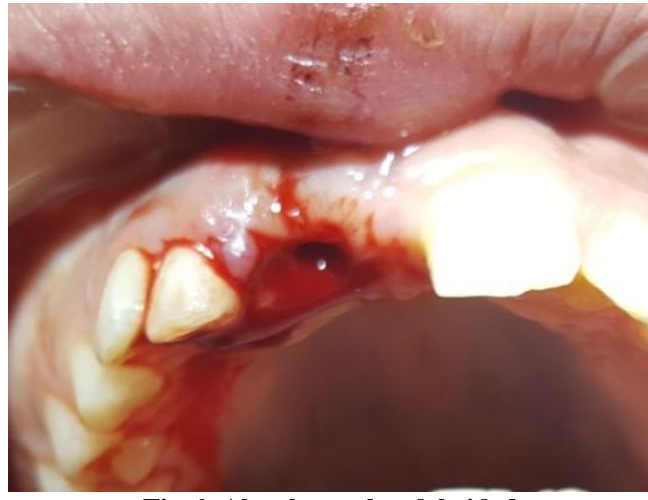

Fig-6: Alveolar socket debrided

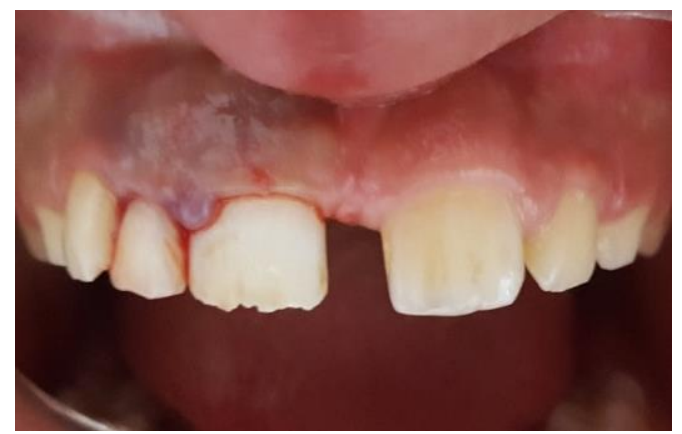

Fig-7: Tooth replanted in socket

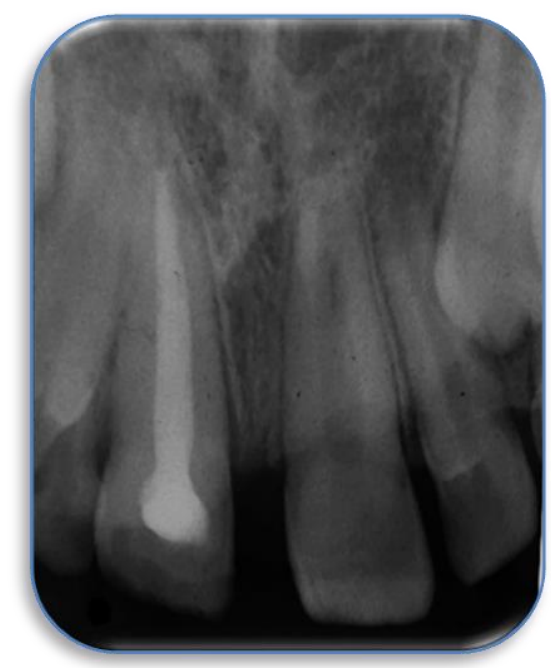

Fig-8: Periapical radiograph showing that the tooth was correctly positioned in the socket

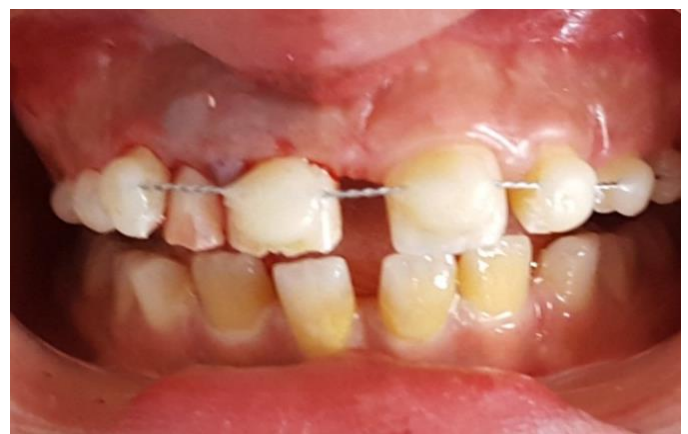

Fig-9: Splinting of the avulsed tooth with orthodontic wire and composite resin 


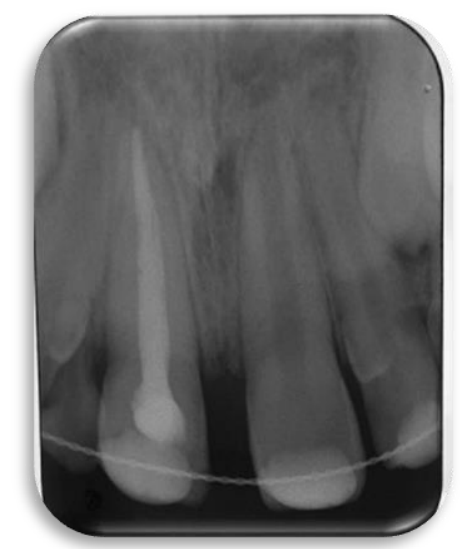

Fig-10: Periapical radiograph after immediate replantation of avulsed tooth

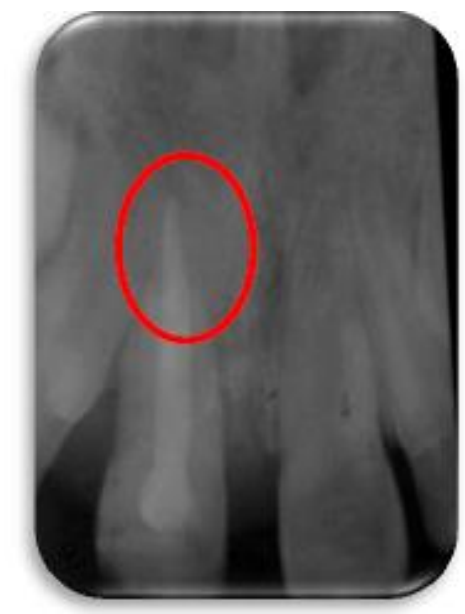

Fig-11: Periapical radiograph after 18 months follow up: Resorption is limited at apical third

\section{DisCUSSION}

Tooth avulsion is considered as an emergency that the dentist must be able to assess rapidly and manage appropriately. Tooth avulsion is characterized by compromised neurovascular supply, pulp necrosis and loss of periodontal ligament cells [7-9].

$\mathrm{N}$ appropriate emergency management and treatment plan are very important for a good prognosis of this injury. The primary goal of avulsed tooth management is to preserve and treat the supporting tooth tissues and to replant the avulsed tooth. The success of replantation depends on the patient's general health, the maturity of the root, the time that the tooth is out of its socket and storage medium. The extraalveolar time and the storage medium have the most critical effect on the status of the periodontal ligament cells $[5,8]$.

Many clinical studies have indicated that the best healing potential of the avulsed tooth is possible if the replantation is performed immediately at the site of injury in order to minimize the extra alveolar time (within $5 \mathrm{~min}$ ). After a dry time of 60 minutes or more, the viability of root surface periodontal ligament cells is unlikely $[3,5,7]$.

The choice of storage for preserving traumatically avulsed tooth is also important for the success of future replantation. Ideally, the tooth should be stored in Hanks Balanced Salt Solution (HBSS), milk, saliva, physiological saline and clean water to keep it clean.

The Hank's Balanced Salt Solution (HBSS) have shown superior ability in maintaining the viability of the periodontal ligament fibers for extended periods. However, they are presently considered to be impractical as they are, except for a few areas of the world, and are not generally available at the accident sites where injury is likely to occur $[2,10]$.

The Pulpal tissue of avulsed tooth with closed apices cannot survive and it must be removed. Endodontic treatment for all avulsed permanent teeth with a closed apex should be initiated $[1,2]$. If the tooth has been dry for more than $60 \mathrm{~min}$ before replantation, the root canal treatment may be done extra-orally prior to replantation or later. Because there were no chances of obtaining pulp space revascularization and the periodontal ligament will be necrotic and not expected to heal $[1,5]$.

In the present case, the avulsed incisor had a closed apex and was kept in milk from the moment of trauma until its replantation $16 \mathrm{~h}$ later.

The use of milk as storage media is recommended by the American Association of Endodontic and it is significantly better than other solutions. Its physiological properties, including $\mathrm{pH}$ and osmolality are compatible to those of the cells from the periodontal ligament. The milk has sufficient nutrients, capable of maintaining the viability of periodontal ligament cells, also it is easy to found it in locations where avulsion accidents $[11,12]$.

In this case, it was decided to treat the root canals extra-orally, as the goal was not immediate replantation considering the prolonged extra oral time of the tooth. The root surface of the avulsed tooth was treated with $2 \%$ sodium fluoride solution for 20 mins before endodontic extra-oral treatment.

Several authors have recommended the use of fluoride solutions in different forms and concentrations to treat the root surfaces in case of delayed tooth reimplantation assuming that demineralized dentin surface would be more prone to fluoride incorporation and might become more resistant to resorption. Fluoride directly acts on the bone tissues, cementum and dentin, by converting hydroxyapatite into fluorapatite [13]. 
For splinting of avulsed teeth, it should allow physiologic movement of teeth during healing and that is in place for a minimal time period as this decreased the incidence of ankylosis [10, 13]. In this case, the tooth was splinted with orthodontic wire and, bonded composite for the following 4 weeks.

The management of this case presented here was in accordance with the accepted replantation protocol described by the International Association of Dental Traumatology [1].

Replanted tooth must be monitored carefully and clinical/radiographical findings should be recorded. In avulsion tooth, damage to periodontal ligament is more severe, hence replacement resorption is evident in most of the cases. Replacement resorption leads to fusion of tooth root with adjacent alveolar bone and resorption may be diagnosed within 2-6 months of reimplantation. This complication occurs by a majority in the first year after replantation, but can also be seen in later periods. The risk of resorption is considerably reduced, if it is not detected within 2 years [5, 13-15].

In this case, the replanted tooth remained in a stable functional position and did not reveal clinical ankylosis or replacement resorption during the 12 months follow-up period, but a replacement root resorption developed later. Therefore, long follow-up period is essential for the replantation cases [15].

\section{CONCLUSION}

Replantation avulsed tooth is the treatment of choice in permanent dentition. Replantation can restore the patient's esthetic appearance and occlusal function and prevent physiological trauma, which may be associated with a missing anterior tooth. According to the presented case, replantation can be advised for avulsed tooth with prolonged extra-oral time, but the risk of resorption at long time should be considered.

If the avulsed tooth had not been replanted, other treatment options might have included prosthetic replacement of the missing incisor, space closure with orthodontic treatment, or autotransplantation of another tooth to the empty space.

\section{REFERENCES}

1. Lars, A., Jens, O.A., Peter, D., Geoffrey, H., Martin, T., Anthony, J., Diangelis, D.J Kenny, A. S., Cecilia, B., Marie, T. F., Morris, L.H., Antonio, R., Lenzi, B. M., Alex, J. M., Mitsuhiro, T. (2012). International Association of Dental Traumatology. Guidelines for the management of traumatic dental injuries: 2. Avulsion of permanent teeth. Dental Traumatology, 28(2), 88-96.
2. Abu-Hussein, M., Watted, N., \& Abdulgani, A. (2014). Replantation of Avulsed Permanent Anterior Teeth: A Case Report. RRJDS, 2(4), 4352.

3. Tezel, H., Atalayin, C., \& Kayrak, G. (2013). Replantation after traumatic avulsion. European journal of dentistry, 7(2), 229.

4. Harris, A., Reshmi, J., George, S., \& Issac, J. S. (2014). Delayed reimplantation: a case report. Journal of international oral health: JIOH, 6(5), 104.

5. Savas, S., Kucukyilmaz, E., Akcay, M., \& Koseoglu, S. (2015). Delayed replantation of avulsed teeth: Two case reports. Case Reports in Dentistry, 2015.

6. Pooja, B., Manohar, P., Sanmati, P.1, Shilpa, S., Farhin, K. (2017). Replantation of an avulsed tooth: a case report, Journal of Dental Health Oral Disorders \& Therapy, 7 (6), 400-404.

7. Verma, L., H.S. (2010). Reimplantation of Avulsed Tooth - A case Study, Journal of Exercise Science and Physiotherapy, 6(2), 126129.

8. Dinesh, R., Supreeth, S., Manipal, S., Upadhyay. (2013). Emergency Management of an Avulsed Tooth, International dental Journal of Student's Research, 1(4), 31- 35.

9. Puri, S. N., Tripathi, S., Pandya, M. B., \& Trivedi, P. R. (2011). Reimplantation of avulsed teeth after dry storage for one week. International Journal of Clinical Dental Science, 2(3).

10. Martin, T. (2011). Avulsion of permanent teeth: theory to practice, Dental Traumatology, 27(4), 281-294.

11. Khinda, V. I., Kaur, G., Brar, G. S., Kallar, S., \& Khurana, H. (2017). Clinical and practical implications of storage media used for tooth avulsion. International journal of clinical pediatric dentistry, 10(2), 158

12. Adnan, S., Lone, M. M., Khan, F. R., Hussain, S. M., \& Nagi, S. E. (2018). Which is the most recommended medium for the storage and transport of avulsed teeth? A systematic review. Dental Traumatology, 34(2), 59-70.

13. Harris, A., Reshmi, J., George, S., \& Issac, J. S. (2014). Delayed reimplantation: a case report. Journal of international oral health: JIOH, 6(5), 104.

14. Day, P. F., Duggal, M., \& Nazzal, H. (2019). Interventions for treating traumatised permanent front teeth: avulsed (knocked out) and replanted.Cochrane database of systematic reviews, (2).

15. Hasanuddin, S., \& Reddy, J. S. (2018). Sequelae of delayed replantation of maxillary permanent incisors after avulsion: A case series with 24month follow-up and clinical review. Journal of Indian Society of Pedodontics and Preventive Dentistry, 36(4), 410. 